\title{
Amazon Ürün Değerlendirmeleri Üzerinde Derin Öğrenme/Makine Öğrenmesi Tabanlı Duygu Analizi Yapılması
}

\author{
Ömer Faruk Göçgün*, Aytuğ Onan² \\ 1* İzmir Katip Çelebi Üniversitesi, Mühendislik Fakültesi, Yazılım Mühendisliği Bölümü, İzmir, Türkiye (ORCID: 0000-0003-1957-0794), farukgocgun@ gmail.com \\ 2 İzmir Katip Çelebi Üniversitesi, Mühendislik Fakültesi, Yazılım Mühendisliği Bölümü, İzmir, Türkiye, (ORCID: 0000-0002-9434-5880), aytug.onan@ikc.edu.tr
}

(2nd International Conference on Access to Recent Advances in Engineering and Digitalization (ARACONF)-10-12 March 2021)

(DOI: $10.31590 /$ ejosat.902674)

ATIF/REFERENCE: Göçgün, Ö. F. \& Onan, A. (2021). Amazon Ürün Değerlendirmeleri Üzerinde Derin Öğrenme/Makine Öğrenmesi Tabanlı Duygu Analizi Yapılması.Avrupa Bilim ve Teknoloji Dergisi, (24), 445-448.

$\ddot{O} z$

Bu çalışma da, amazon ürün yorumları veri seti üzerinde Makine öğrenmesi yöntemleriyle, duygu analizi sınıflandırma performansları analiz edilmiştir. Yapılan çalışma, ürün yorumlarının duygu içerip içermediği, içeriyorsa olumlu, olumsuz veya tarafsız olma durumunun belirlenmesidir. Çalışmada, Kaggle amazon ürün yorumları[1] içeren veri seti edinilmiş ve Python dili kullanılarak Jupyter Lab üzerinde 4 farklı makine öğrenmesi ile sistem çalıştırılmıştır. Çapraz doğrulama yöntemi $\mathrm{k}=10$ olacak şekilde kullanılmıştır. Kullanılan yöntemler içerisinde Karar Ağacı sınıflandırma algoritması kullanılan diğer Naive Bayes, Destek Vektör Makineleri ve Lojistik Regresyon yöntemlerine göre yüzde 94 doğruluk oranı ile daha başarılı olduğu deneysel olarak gösterilmiştir.

Anahtar Kelimeler: Duygu analizi, Makine öğrenmesi, F1-puanı.

\section{Sentiment Analysis with Deep Learning/Machine Learning Techniques on Amazon Products Review}

\begin{abstract}
In this study, emotion analysis classification performances of comments were analyzed using machine learning methods on amazon product reviews data set. The study conducted is the determination of whether the product comments contain emotions, if comment contains emotions, whether they are positive, negative or neutral. In the study, the data set includes Kaggle Amazon product reviews [1] was obtained and the system was run with 4 different machine learning algoritms using Python language on Jupyter Lab. The cross validation method was used as $\mathrm{k}=10$. Among the methods used, it has been experimentally indicates that using Logistic Regression classification algorithm is more successful with an accuracy rate of 96 percent compared to other Naive Bayes, Support Vector Machines and Decision Tree methods
\end{abstract}

Keywords: Sentiment Analysis, Machine learning, F1-Score.

\footnotetext{
*Sorumlu Yazar: farukgocgun@gmail.com
} 


\section{Avrupa Bilim ve Teknoloji Dergisi}

\section{Giriş}

Makine Öğrenmesi(MÖ), öğrenme işlemini önceki gözlemlere veya deneyimlere dayanarak geliştirilen sistemlerin geliştirilmesidir[2]. Görüş madenciliği olarak da bilinen duygu analizi ile karar verme sürecini etkileyen duygular ve görüş pazar analizi ve müşteri memnuniyeti gibi pek çok konunun tahmin edilebilmesi sağlanabilmektedir[3]. Duygu analizi ile metinlerde geçen duygusal kelimelerin çıkartılması ve analiz edilen yazının olumlu, olumsuz veya tarafsız olma durumunun belirlenmesi hedeflenir.[4]

Amazon ürün yorumları üzerinde duygu analizini gerçekleştirmek için pek çok makine öğrenmesi çalışmaları bulunmaktadır.

W. Tan, X. Wang ve X. Xu (2018) çalışmalarında, Naive Bayes, destek vektör makineleri, $\mathrm{k}$ en yakın komşu ve yenilenen sinir ağları sınıflandırıcılarının Amazon ürün yorumları üzerinde duygu analizi için tahmin etme başarımlarını değerlendirmiştir. Bu çalışmada, yapay sinir ağları için kullanılan Uzun Kısa Süreli Bellek (LTSM)yöntemiyle \%71.5 ile doğru sınıflandırma elde edildiği gözlenmiştir[5].

J. Sadhasivam ise Naive Bayes, destek vektör makineleri ve ensemble yaklaşımıyla Amazon ürün yorumları üzerinde duygu analizi için başarımları değerlendirmiş ve ensemble yaklaşımıyla \%78 civarında başarım elde ettiği gözlenmiştir[6].

\section{Materyal ve Metot}

\subsection{Materyal}

Kaggle[1] üzerinden çekilen veri, ön işlemlerden geçirildikten sonra sınıflandırma algoritmaları ile duygu analizi gerçekleştirilerek çıkan sonuçlar karşılaştırılmıştır.

\subsubsection{Veri Seti}

Amazon tüketici ürün yorumları üzerinde duygu analizi yapılabilmesi için Kaggle[1] üzerinde bulunan veri setleri indirilmiş ve en güncel olan (Products_May2019) veri seti kullanılmıştır.

\subsubsection{Veri Özellikleri}

28332 satır ve 24 adet kolondan oluşan veri setimizde, oy ve yorum kolonları haricinde ki tüm kolonlar silinmiştir. Yorumlara verilen oyların grafiği şekil 1'de sunulmuştur.

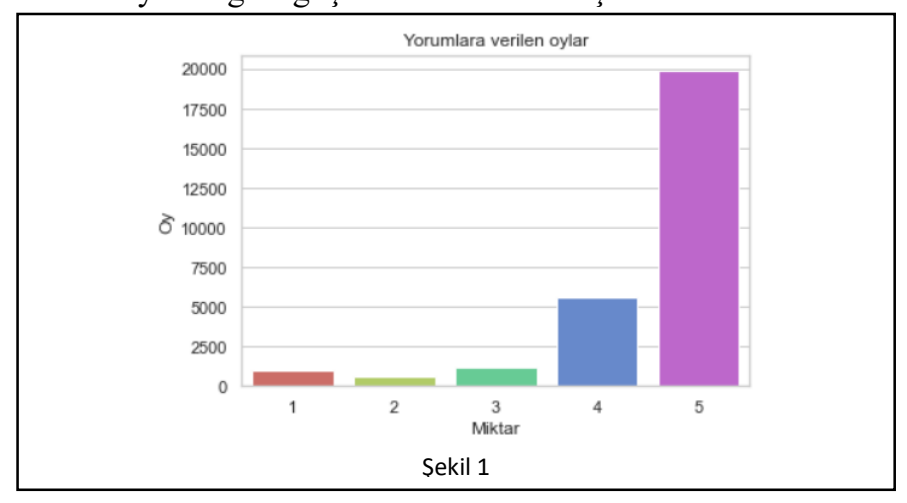

\subsection{Metod}

$\mathrm{Bu}$ çalışmada kullanılan veri seti, verinin ön işlenmesi ve analizi, duygu sınıflandırması ve görselleştirmesi sunulmaktadır. Yapılan çalışmada doğruluk, F1 Skor ve hata oranı metrikleri kullanılmıştır.

Uygulamaların gerçekleştirilmesinde, Python programlama dili tercih edilmiş. Kodlama ortamı olarak Jupyter Lab kullanılmıştır.

Python, Guido van Rossum tarafından ilk sürümü 1991'de ortaya konan genel amaçlı bir programlama dilidir. Diğer dillere göreli öğrenim kolaylığı ve geniş kütüphane desteğiyle oldukça yaygın kullanıcı kitlesine ulaşmıştır.[7] PYPL programlama dilleri popülerlik indeksi 'ne göre Aralık 2019-Aralık 2020 popülerlik sıralamasında Python dilinin 1. Sırada olduğu gözlemlenmiştir[8].

Kodlamalar için Jupyter Lab Notebook kullanılmıştır. Jupyter Lab, çeşitli programlama dilleri için etkileşimli, not defteri benzeri basit bir web ara yüzü bulunan kodları okuyup çalıştırmayı bloklar halinde yapabilen oldukça kullanıcı dostu bir uygulamadır.

Natural Language Toolkit NLTK, insan dili verileriyle çalışmak üzere Python programları oluşturmak için geliştirilmiş platformdur. Sınıflandırma, belirteçleştirme, kök belirleme, etiketleme, ayrıştırma ve anlamsal akıl yürütme için metin işleme[9].

\subsubsection{Veri Önişleme}

Yorumların analiz edilebilmesi ve sinıflandırmayı daha verimli olması açısından yorumlar çeşitli önişlemlerden geçirilmiştir.

Önişleme işlemlerinde, belirteçleştirme, kök belirleme, etiketleme, sık kullanılan önemsiz kelimelerin çıkartılması ve tüm harfleri küçük harfe dönüştürme gibi işlemler yapılmıştır.

1 ile 5 arası verilen oylar 3 için tarafsız( 0 değeri), 3'ten küçük için olumsuz(-1 değeri) ve 3 'ten büyük için olumlu(+1) duygu içerdiğine ilişkin oyların duygu değerleri $(-1,0$ ve +1$)$ hesaplanarak yeni bir kolonda sonuçlar kayıt altına alınmış ve Tablo 1'de gösterilmiştir.

Tablo 1. Duygu Değerlendirmeleri Kayıt Sayıları

\begin{tabular}{l|l}
\hline Veri(Duygu) & Kayit Sayısı \\
\hline+1 (Olumlu) & 25545 \\
\hline-1 (Olumsuz) & 1581 \\
\hline 0 (Tarafsiz) & 1206 \\
\hline
\end{tabular}

Çalışmada, her bir verideki kelimeler birbirinden ayrıştırılmıştır, ayrıştırma işlemi sonucunda örnek veri şu şekildedir:[ 'I', 'order', '3', 'of', 'them', 'and', 'one', 'of', 'the', 'item', 'is', 'bad', 'quality', '.', 'Is', 'missing', 'backup', 'spring', 'so', 'I', 'have', 'to', 'put', 'a', 'pcs', 'of', 'aluminum', 'to', 'make', 'the', 'battery', 'work', '.']

Doğal Dil İşleme Kütüphanesi olan NLTK Kütüphanesi ve Etkisiz Kelimeler (stopwords) ile Kelimelerin önek ve 
soneklerini eklenmiştir.

ayrıştırabilmek için PorterStemmer paketi

Veri setindeki tüm yorumlar bir döngü içerisinde gereksiz kelimelerden ve kelimelerin önek ile soneklerinden temizlenmiştir.

Metin sınıflandırma işlemleri için kelimenin metinde geçme sıklığı TF-IDF (Term Frequency-Inverse Document Frequency) yöntemiyle metnin sayısal veriye dönüştürülmesini sağlanmıştır.

Ön işleme sonuçları yeni bir veri seti olarak kayıt altına alınmıştır.

\subsubsection{Kullanılan Sinıflandırma Algoritmaları}

Bu çalışma kapsamında, tüketici yorumlarının duygu analizi için 4 farklı sınıflandırma kullanılmıştır. Bunlar Naive Bayes(NB) sınıflandırıcısı, destek vektör makineleri(SVM), karar ağacı(DT ve lojistik regresyon (LR) algoritmalarıdır.

\subsubsection{Naive Bayes (NB)}

Temelinde bağımsız değişken varsayımlarını (ilgili sınıfa ait bir öznitelik bir diğerinden bağımsız olduğu) konu alan Bayes teoremi, basit ve kullanımı kolay makine öğrenme algoritmalarından biri [11] olmasına rağmen yüksek başarımı nedeniyle sıklıkla uygulanmaktadır [3].

\subsubsection{Destek Vektör Makineleri(SVM)}

Destek vektör makinesi iki boyutlu uzayda doğrusal, üç boyutlu uzayda düzlemsel ve çok boyutlu uzayda hiper düzlem şeklindeki ayırma mekanizmaları ile veriyi iki ya da daha çok sınıfa ayırma yeteneğine sahiptir [12].

\subsubsection{Karar Ağaçları(DT)}

Karar ağaçları gözetimli öğrenme sınıflandırmasında yaygın olarak kullanılan bir algoritma türüdür. Böl ve yönet yaklaşımında yararlanan bir öğrenme modeli, verimli bir şekilde sınıflandırma ve regresyon gerçekleştiren bir yöntemdir. Karar Ağaçları basit ve anlaşılır bir yapıya sahiptir [13].

\subsubsection{Lojistik Regresyon (LR)}

Lojistik regresyon, özellikler arasındaki ilişkiyi açıklayan, sınıf etiketi $\{0,1\}$ şeklinde verilen ikili sınıflandırma problemlerinde kullanılan sınıflandırma yöntemidir[13].

\subsubsection{Sınıflandırma Algoritmalarının Karşılaştırılmasında Kullanılan Kriterler}

Modellerin sinıflandırma performansları, F-1 puanı, kesinlik (precision), duyarlılık (recall) ve doğruluk (accuracy) metrikleri ile sunulmuş ve sınıflandırma performanslarının geçerliliğini artırmak için, $\mathrm{k}=10$ katlamalı çapraz doğrulama yöntemiyle test işlemi tekrar ortaya gerçekleştirilmiştir.

\subsubsection{K katlamalı çapraz doğrulama}

Çapraz doğrulama, tahmine dayalı modellerin genelleme yeteneğini değerlendirmek ve gereğinden fazla uyum ya da yetersiz uyum göstermeyi önlemek için bir veri yeniden örnekleme yöntemidir[10]. K-n katlamalı çapraz doğrulama (K- fold Cross-Validation) örneklem verilen $\mathrm{n}$ değeri kadar doğrulama işlemi tekrar edilir. $\mathrm{Bu}$ çalışmada $\mathrm{k}=10$ değeri verilmiştir. 10 defa tekrar yapılan doğrulama da ağırlıklı ortalama alınarak doğruluk oranını daha verimli olması hedeflenmiştir.

\subsubsection{Doğruluk}

Bir sınıflandırıcının doğruluğu (Accuracy), toplam doğru sınıflandırılmış pozitiflerin bölünmesi ve toplam numune sayısına göre negatifler ile hesaplanmaktadır [15]:

$$
\text { Doğruluk }=\frac{T N+T P}{T P+F P+F N+T N}
$$

\subsubsection{Hassasiyet}

Bir sınıflandırıcının hassasiyeti (Recall), hassasiyet değeri pozitif olarak tahminlenmesi gereken değerlendirmelerin ne kadarının pozitif olarak tahmin ettiğimizi gösteren bir metriktir. [15]

$$
\text { Hassasiyet }(R E C)=\frac{T P}{T P+F N}
$$

\subsubsection{Kesinlik}

Duyarlılık (Precision) değeri pozitif olarak tahminlenmesi gereken değerlendirmelerin ne kadarının pozitif olarak tahmin ettiğimizi gösteren bir metriktir. [15]

$$
\operatorname{Kesinlik}(P R E)=\frac{T P}{T P+F P}
$$

\subsubsection{F-Ölçütü(F-Measure)}

F-Ölçütü, Kesinlik (precision) ve duyarlılık (recall) değerlerinin harmonik ortalamasını göstermektedir. [15]

$$
F-\text { measure }=\frac{2 * P R E * R E C}{P R E+R E C}
$$

Formüllerde belirtilen; TP, $F P, T N$ ve $F N$ sırası ile doğru pozitif, yanlış pozitif, doğru negatif ve yanlış negatif sayılarını temsil etmektedir [15].

\subsubsection{ROC eğrisi ve Eğri Altında Kalan Alan}

ROC eğrisi, sınıflandırma performansını gösterir. $\mathrm{X}$ ekseninde yanlış pozitif oran(FPR) ve Y ekseninde doğru pozitif oran (TPR) bulunmaktadır.

AUC, "ROC Eğrisi Altındaki Alan" anlamına gelir. Yani, AUC, $(0,0)$ 'dan $(1,1)$ ' e kadar tüm ROC eğrisinin altındaki iki boyutlu alanın tamamını ölçerek, tüm olası sınıflandırma eşiklerinde toplu bir performans ölçüsü sağlar.[16]

\section{Araştırma Sonuçları ve Tartışma}

Bu çalışmada 28332 adet ürün yorumu içeren veri seti kullanılmıştır. Makina öğrenmesi yöntemleriyle Naive Bayes (NB), Destek Vektör Makineleri (SVM), Karar Ağacı (DT) ve Lojistik Regresyon (LR) sinıflandırma algoritmalarından yararlanılmıştır. Veri setinin eğitimi ve sınıflandırma sürecinden önce ön işleme aşamaları uygulanmıştır. Ön işlemeden sonra yorumları içeren metnin sayısal veriye dönüştürülmesi için kelimenin metinde geçme sılı̆̆ı(TF-IDF) yöntemi kullanılmıştır. Sınıflandırma algoritmalarının hepsinde ver setinin \%80’i eğitim, \%20'si ise test için kullanılmıştır. 


\section{Sonuç}

Kullanılan yöntemler içinde Karar ağacı yöntemi \%94 doğruluk oranı ve \%75 F-puanıyla yüksek başarım elde etmiştir. Diğer sınıflandırıcılara göre Naive Bayes daha düşük başarım elde etmiştir.

Şekil 2'de ise sınıflandırıcıların eğri altında kalan(AUC) grafiği gösteriliştir.

Tablo 2.Sinıflandirıcıları Analiz Oranlart

\begin{tabular}{|l|c|c|c|c|}
\hline \multirow{2}{*}{ Test sonuçları } & \multicolumn{4}{|c|}{ Sinıflandırma Algoritmaları } \\
\cline { 2 - 5 } & NB & SVM & DT & LR \\
\hline 1) Doğruluk & 0.90 & 0.93 & $\mathbf{0 . 9 4}$ & 0.92 \\
\hline $\begin{array}{l}\text { 2) Katlamalı çapraz } \\
\text { doğrulama }\end{array}$ & 0.90 & 0.92 & $\mathbf{0 . 9 3}$ & 0.92 \\
\hline 3)Kesinlik & 0.86 & 0.90 & $\mathbf{0 . 7 7}$ & 0.71 \\
\hline 4) Hassasiyet & 0.37 & 0.54 & $\mathbf{0 . 7 4}$ & 0.47 \\
\hline 5) F-1 Puanı & 0.39 & 0.57 & $\mathbf{0 . 7 5}$ & 0.51 \\
\hline 6) AUC & 0.93 & 0.95 & $\mathbf{0 . 9 6}$ & 0.94 \\
\hline
\end{tabular}

Şekil 2.ROC-AUC Değerleri

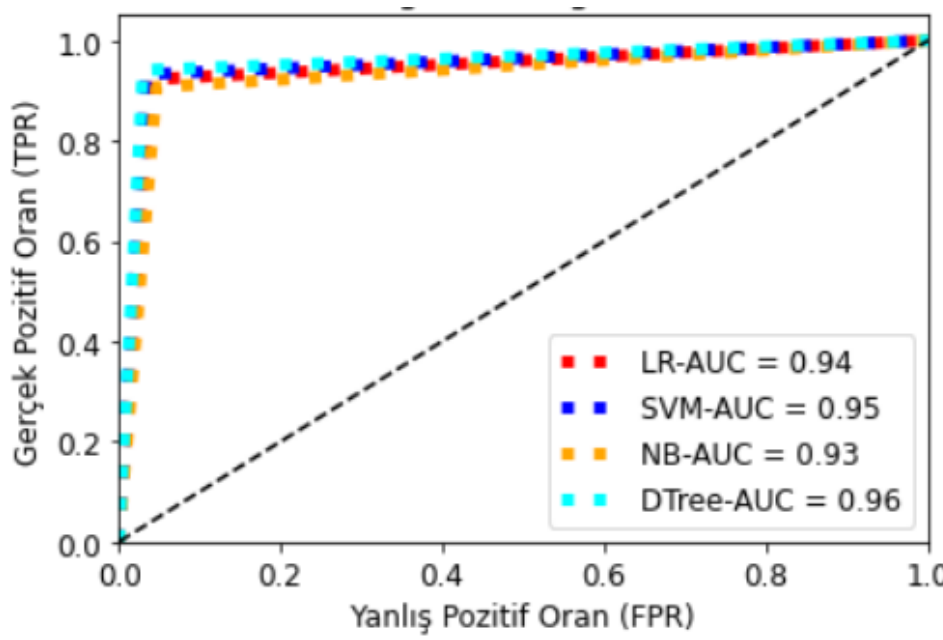

\section{Kaynakça}

[1] Amazon Tüketici Ürün Değerlendirmeleri veri seti, Erişim adresi https://www.kaggle.com/datafiniti/consumerreviews-of-amazon-products

[2] Bilgin M. (2017), "Gerçek Veri Setlerinde Klasik Makine Öğrenmesi Yöntemlerinin Performans Analizi”, 19 Akademik Bilişim Konferansı Breast, 2(9) 683.

[3] Onan, A. (2017). Twitter mesajları üzerinde makine öğrenmesi yöntemlerine dayalı duygu analizi. Yönetim Bilişim Sistemleri Dergisi, 3 (2) , 1-14. Erişim adresi https://dergipark.org.tr/tr/pub/ybs/issue/33128/368593

[4] Seker S.E. (2015), "Metin madenciliğii”, YBS ansiklopedi, 2(3), 32. Erişim adresi http://ybsansiklopedi.com/wpcontent/uploads/2015/08/MetinMadenciligi30_32.pdf

[5] Tan W, Wang X. ve Xu X.(2018), “Analysis for Amazon Reviews" Erişim adresi http://cs229.stanford.edu/proj2018/report/122.pdf
[6] Sadhasivam, Jayakumar \& Babu, Ramesh. (2019). Sentiment Analysis of Amazon Products Using Ensemble Machine Learning Algorithm. International Journal of Mathematical, Engineering and Management Sciences. 4. 508-520. 10.33889/IJMEMS.2019.4.2-041.

[7] Malkoç B.(2012), "Temel Bilimler ve Mühendislik Eğitiminde Programlama Dili Olarak Python”, XIV. Akademik Bilişim Konferansı Bildirileri, 201.

[8] PYPL, programlama dilleri popülerlik indeksi(2020), Erişim Adresi https://pypl.github.io/PYPL.html

[9] NLTK (2020) Erişim Adresi http://www.nltk.org/

[10]Berrar D.(2019), “Cross-validation”, Encyclopedia of Bioinformatics and Computational Biology 1 (542-545)

[11] Şahin E. (2018), "Makine öğrenme yöntemleri ve kelime kümesi tekniği ile istenmeyen eposta sınıflandırması" yüksek lisans tezi, sayfa 8, Hacettepe üniversitesi, Bilgisayar Mühendisliği Anabilim Dalı.

[12] Güran, A, Uysal, M, Doğrusöz, Ö. (2014). Destek vektör makineleri parametre optimizasyonunun duygu analizi üzerindeki etkisi. Dokuz Eylül Üniversitesi Mühendislik Fakültesi Fen ve Mühendislik Dergisi, 16 (48) , 86-93. Erişim adresi https://dergipark.org.tr/tr/pub/deumffmd/issue/40797/492168

[13] Kalayc1, T. (2018). Kimlik hırsızı web sitelerinin sınıflandırılması için makine öğrenmesi yöntemlerinin karşılaştırılması. Pamukkale Üniversitesi Mühendislik Bilimleri Dergisi, 24 (5) , 870-878. Erişim Adresi https://dergipark.org.tr/tr/pub/pajes/issue/39683/469468

[14] Yengi, Y, İlhan Omurca, S. (2016). Lojistik Regresyonun Özellik Azaltma Teknikleri ile Gen Dizilimlerinin Sınıflandırılmasındaki Başarısı. Türkiye Bilişim Vakfı Bilgisayar Bilimleri ve Mühendisliği Dergisi, 8 (1) , 1-12. https://dergipark.org.tr/tr/pub/tbbmd/issue/22248/238832

[15] Memiş, S, Enginoğlu, S, Erkan, U. (2019). A Data Classification Method in Machine Learning Based on Normalised Hamming Pseudo-Similarity of Fuzzy Parameterized Fuzzy Soft Matrices. Bilge International Journal of Science and Technology Research, ICONST 2019, 1-8. DOI: 10.30516/bilgesci.643821

[16] Google Machine Learning Crash Course(2020), Erişim adresi https://developers.google.com/machinelearning/crash-course/classification/roc-and-auc 